\title{
A Narrative Review of Gut-Muscle Axis and Sarcopenia: The Potential Role of Gut Microbiota
}

This article was published in the following Dove Press journal:

International Journal of General Medicine

Jiaxi Zhao (D)

Yiqin Huang

Xiaofeng Yu

Huadong Hospital Affiliated to Fudan University, Shanghai, People's Republic of China
Correspondence: Xiaofeng $\mathrm{Yu}$

Tel +86 I8I 21227529

Email yuxiaofeng252@163.com
Abstract: Sarcopenia is a multifactorial disease related to aging, chronic inflammation, insufficient nutrition, and physical inactivity. Previous studies have suggested that there is a relationship between sarcopenia and gut microbiota,namely, the gut-muscle axis. The present review highlights that the gut microbiota can affect muscle mass and muscle function from inflammation and immunity,substance and energy metabolism, endocrine and insulin sensitivity, etc., directly or indirectly establishing a connection with sarcopenia, thereby realizing the "gut-muscle axis".

Keywords: gut microbiota, metabolism, inflammation, muscle

\section{Introduction}

Recent studies put forward a relationship between sarcopenia and gut microbiota, namely, the gut-muscle axis. Although some of them explain this relationship from certain angles, most of them are one-sided and ignoring several possible special influencing factors, such as immunity and endocrine. This article will explain the gut-muscle axis in detail from the following aspects: inflammation, immunity, substance and energy metabolism, endocrine and insulin sensitivity, etc., and reveal how different kinds of Nutritional Supplements affect muscle function and mass through the gut microbiota. List the facts that different types of exercise can have different effects on muscles and gut microbiota. The following content will introduce sarcopenia and gut microbiota separately.

\section{Introduction to Sarcopenia}

Sarcopenia is an involuntary loss of skeletal muscle mass and strength. ${ }^{1}$ It is a multifactorial disease rated to aging, chronic inflammation, insufficient nutrition, physical inactivity, and endocrine system disorders (such as reducing estrogen and androgens). So far, several diagnostic criteria for sarcopenia, determined by different races and different regions, have been proposed. ${ }^{1-3}$ Working Group of the International Sarcopenia Conference, EWGSOP2 (European Working Group on Sarcopenia in Older People), and AWGS2019 (Asian Working Group for Sarcopenia 2019), The European Working Group on Sarcopenia in Older People (EWGSOP) established its first version of sarcopenia's diagnostic criteria in 2010, which was then further revised in 2018. Today, the definition of sarcopenia was mainly based on the decrease in muscle mass and muscle function. ${ }^{4,5}$ The muscle function is commonly evaluated using handgrip strength, 5-times repeated chair stand test, and 4-meter walking speed. ${ }^{1}$ Grip strength is closely related to lower 
limb muscle strength and calf muscle cross-sectional area; thus, this test has been recommended as the golden approach for diagnosis and prognosis prediction. ${ }^{6,7}$

In a large cross-sectional study, 973 participants (from a total of 11253 subjects) were diagnosed with sarcopenia using EWGSOP2, and the incidence of sarcopenia significantly increased with age. ${ }^{8}$ Another cross-sectional study in the Korean community showed that among 2099 community older adults aged 70-84, sarcopenia incidence was $4.6-14.5 \%$ in men and $6.7-14.4 \%$ in women. ${ }^{9}$ In general, the reduction of muscle mass and strength in sarcopenia is closely related to aging. In addition, this systemic skeletal muscle disease increases the risk of falls in the elderly, prolongs the length of hospitalization and costs, and increases morbidity and mortality. Compared with the higher arm muscle cross section $(\geq 33.4 \mathrm{~cm} 2)$, the lower arm muscle cross section $(<23.5 \mathrm{~cm} 2)$ has a higher risk of death (HR 2.03; 95\% CI, 1.36-3.02). Compared with those without sarcopenia, the risk of falls in men with sarcopenia was higher (OR 2.58; 95\% CI, 1.42-4.73). ${ }^{10,11}$

\section{Introduction to Gut Microbiota}

The gut microbiota can affect the host's muscle mass and function by regulating systemic inflammation and immunity, substance and energy metabolism, and insulin sensitivity. With the development of high-throughput sequencing technology and bioinformatics technology, ${ }^{12}$ so far, over 9.9 million microbial genes have been detected in human feces, ${ }^{13}$ including more than 1000 different bacteria, ${ }^{11}$ most of which are anaerobes. ${ }^{14}$ Probiotics are beneficial bacteria (eg, Lactobacillus, Bifidobacterium, Clostridium butyricum, Bacillus subtilis) that are primarily found in our digestive system. And prebiotics are mainly oligosaccharides that cannot be digested and absorbed by the host ${ }^{15}$ that feed other beneficial bacteria, promoting their growth and reproduction. The preparation that mixes probiotics and prebiotics are called synbiotics. ${ }^{16}$ Synbiotics unify the benefits of probiotics and prebiotics, and have been widely used in related experiments.

Gut microbiota changes with age. Healthy aging is related to changes in the structure of the gut microbiota, ${ }^{17,18}$ and its imbalance has a close relationship with human health and disease. Najmeh Seifi discovered that supplementing the synbiotics for critically ill patients can shorten the length of ICU stay, reduce muscle protein catabolism, and reduce infection complications. ${ }^{19}$

This review highlighted the relationship between sarcopenia and gut from the perspective of gut microbiota, namely, the gut-muscle axis. We found that the gut microbiota can affect muscle mass and muscle function from inflammation, immunity, substance and energy metabolism, endocrine and insulin sensitivity, etc., directly or indirectly establishing a connection with sarcopenia, thereby realizing the "gut-muscle axis". The next content will introduce in detail.

\section{Search Strategy}

We searched through PubMed, Scopus, Web of Science, EMBASE databases. First, a basic search was done in PubMed, then the same strategy was applied to other databases. Articles published before 1st October, 2020 were searched using the following key terms: microbiota AND muscle; microbiota AND sarcopenia; microbiota AND "muscle mass"; microbiota AND" muscle strength"; microbiota AND "muscle function". Two researchers searched the above keywords separately and removed duplicate documents. We chose to include experimental and observational studies, including animal and human experiments. In order to avoid the limitations of English, we also expanded the search scope to Chinese. However, there are some articles in other languages that cannot be fully searched. A total of 178 articles were retrieved after screening.

\section{The Potential Role of Gut Microbiota in Gut-Muscle Axis Phenomenon: Gut Microbiota with Muscle Function/Muscle Mass}

So far, a number of studies, including in vitro experiments and preclinical studies, have directly or indirectly proved a certain relationship between muscle mass and gut microbiota. Table 1. For example, Fielding et al transferred fecal samples from two older adults (HF (high-functioning groups) and LF (low-functioning groups)) into germ-free mice. The grip strength was significantly increased in HF, when compared with LF mice. ${ }^{20}$ A randomized controlled double-blind study found that prebiotic increases the grip strength in elderly people over 65 years old. ${ }^{21}$ There was no significant difference in grip strength between the two groups before the intervention, but after 13 weeks of prebiotic supplementation, compared with the placebo group, the grip strength of the experimental group was significantly increased $(10.2 \pm 4.1 \mathrm{~kg}$ vs $12.4 \pm 3.2 \mathrm{~kg}, \mathrm{p}<0.05)$. Munukka et al found that compared with controlled group, supplement of prebiotic (Faecalibacterium prausnitzi) can 
Table I Existing Facts of Animal and Human Experiments: The Effects of Intestinal Microbiota on Muscle Function and Quantity

\begin{tabular}{|c|c|c|c|c|}
\hline References & Objects & Methods & Results & Mechanisms \\
\hline $\begin{array}{l}\text { Fielding } \\
\text { et } \mathrm{al}^{20}\end{array}$ & $\begin{array}{l}\text { Germ- } \\
\text { free mice }\end{array}$ & $\begin{array}{l}\text { Transferred fecal samples from two } \\
\text { older adults (high-functioning groups } \\
\text { and low-functioning groups) into } \\
\text { germ-free mice }\end{array}$ & $\begin{array}{l}\text { HF-colonized mice has } \\
\text { stronger grip strength } \\
\text { compared with LF- } \\
\text { colonized mice }\end{array}$ & Strengthened the intestinal barrier \\
\hline Chen et $\mathrm{al}^{3 !}$ & $\begin{array}{l}\text { Older } \\
\text { people } \\
\text { aged } 65 \\
\text { and over }\end{array}$ & $\begin{array}{l}\text { Supplementation of probiotics: L. } \\
\text { plantarum TWK IO (LPI0) }\end{array}$ & $\begin{array}{l}\text { Improvement of muscle } \\
\text { strength and endurance }\end{array}$ & $\begin{array}{l}\text { Reduced cytokine production; enhanced } \\
\text { glucose utilization }\end{array}$ \\
\hline Lee et $\mathrm{al}^{23}$ & Mice & $\begin{array}{l}\text { Supplementation of Lactobacillus } \\
\text { salivarius }\end{array}$ & $\begin{array}{l}\text { Improvement of muscle } \\
\text { strength and endurance }\end{array}$ & $\begin{array}{l}\text { Related to the increased BCAA pathway, } \\
\text { BCAA can increase } \\
\text { themicroorganismMethanobrevibactersmithii, } \\
\text { which can use } \mathrm{H} 2 \text { in the colon to convert into } \\
\text { SCFA and ATP,thereby providing energy for } \\
\text { synthesis of muscle protein. }\end{array}$ \\
\hline Lee et $\mathrm{al}^{24}$ & Mice & $\begin{array}{l}\text { Supplementation of Bifidobacterium } \\
\text { longum }\end{array}$ & $\begin{array}{l}\text { Increase of muscle } \\
\text { strength, and endurance }\end{array}$ & Increased intestinal SCFA content \\
\hline $\begin{array}{l}\text { Kazmierczak- } \\
\text { Siedlecka } \\
\text { et } \mathrm{al}^{28}\end{array}$ & $\begin{array}{l}\text { Healthy } \\
\text { people } \\
\text { and } \\
\text { cancer } \\
\text { patients }\end{array}$ & $\begin{array}{l}\text { Supplementation of lactobacillus } \\
\text { (extracted from pickle) }\end{array}$ & $\begin{array}{l}\text { Improvement of exercise } \\
\text { performance, reduction } \\
\text { of the muscle loss in } \\
\text { cancer patients }\end{array}$ & $\begin{array}{l}\text { Increased iron absorption, reduced the side } \\
\text { effects of antibiotics on colonic fermentation }\end{array}$ \\
\hline $\begin{array}{l}\text { Munukka } \\
\text { et } \mathrm{al}^{22}\end{array}$ & Mice & $\begin{array}{l}\text { Supplement of Faecalibacterium } \\
\text { prausnitzi }\end{array}$ & Increase of muscle mass & $\begin{array}{l}\text { Enhanced mitochondrial respiration; reduced } \\
\text { inflammation; improved insulin sensitivity }\end{array}$ \\
\hline
\end{tabular}

Abbreviations: BCAA, branched chain amino acid; SCFA, short-chain fatty acids; ATP, adenosine triphosphate.

increase muscle mass (95g vs $110 \mathrm{~g}, \mathrm{p}<0.05)$ by enhanced mitochondrial respiration; reduced inflammation and improved insulin sensitivity. ${ }^{22}$

Over recent years, probiotics have been increasingly used in athletes because of their improved performance and reduced fatigue after exercise. Lee et al studied the effects of Lactobacillus salivarius in mice, revealing that a 4-week supplementation of Lactobacillus could significantly improve muscle strength and endurance, increase liver and muscle glycogen storage, blood lactic acid, BUN, and acid kinase after exercise. ${ }^{23}$ Furthermore, Lee et al transferred Bifidobacterium longum subsp and Bifidobacterium longum OLP-01 (extracted from the intestines of the 2008 women's $48 \mathrm{~kg}$ weightlifting gold medal champion) into the mice. After 4 weeks of treatment, glycogen, liver glycogen content, muscle strength, and endurance all increased. ${ }^{24}$ Moreover, after antibiotic treatment, the mice's exercise endurance was greatly reduced, which was related to the muscle's ability to use glycogen for energy production. ${ }^{25}$

In nature, fermented food is an important source of probiotics, which contain thousands of live lactic acid bacteria (mainly lactobacillus). ${ }^{26,27}$ KazmierczakSiedlecka et al found that lactobacillus (extracted from pickle) supplementation could improve exercise performance in a dose-dependent manner and significantly increases muscle mass in healthy people, and reduce the muscle loss in cancer patients. ${ }^{28}$ These experiments suggest that lactobacillus may become a biological agent for improving physiological function. $^{29}$

In patients with cancer and chronic wasting disease, the balance of gut microbiota is of great significance. Supplementing the corresponding microbiota is also beneficial to fight the disease. ${ }^{28,30}$ Therefore, probiotics are closely related to muscle strength and function and muscle mass. Although there is limited understanding of probiotics' mechanism on muscle strength and quality benefits at this stage, it is clear that gut microbiota is linked to muscles through endocrine, substance and energy metabolism, immunity and inflammation. The possible mechanisms of gut-microbiota are summarized in Figure 1. 


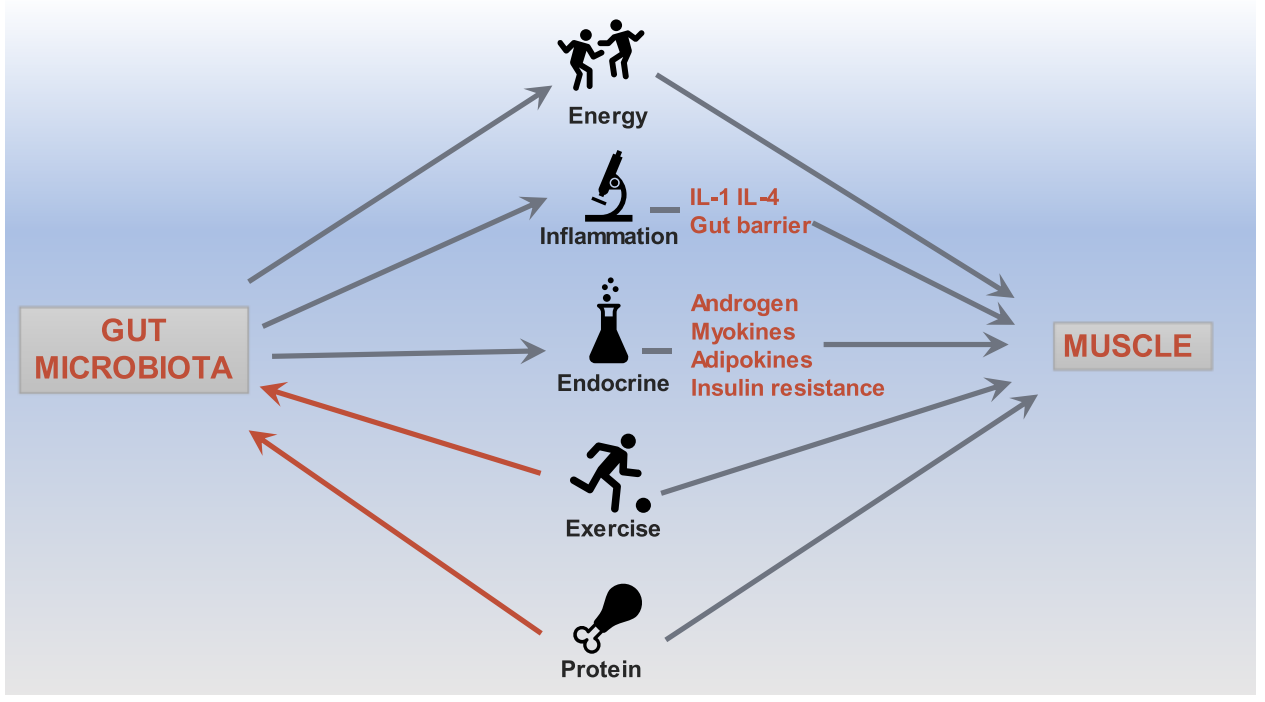

Figure I Overview of the main mechanisms of gut-muscle axis.

\section{Mechanism: Mitochondrial and Energy Metabolism}

Skeletal muscle mitochondrial dysfunction or decline is thought to be one of sarcopenia causes. ${ }^{32}$ Muscle mitochondrial function and content, as well as resting and maximal oxygen (O2) consumption, decrease with age. $^{33,34}$ As age increases,abnormally enlarged mitochondrial fragments can be seen under the electron microscope. ${ }^{35}$ ATP that needed for muscle protein synthesis decreases. ${ }^{36}$ Consequently, maintaining healthy mitochondrial mass has a vital role in combating aging-related muscle atrophy and weakness. ${ }^{37}$

Gut microbiota is connected with skeletal muscle mitochondria level through the production of insulin-like growth factor 1 (IGF-1). ${ }^{38}$ Lahiri et al compared the skeletal muscle of germ-free mice that lacked a gut microbiota to the skeletal muscle of pathogen-free mice with gut microbiota and found muscle atrophy decreased IGF1 , and decreased mitochondrial function in germ-free mice without microbiota. After transplanting the microbiota in the gut, the muscle atrophy and the muscle's antioxidant capacity were significantly improved. ${ }^{39}$ The liver mainly synthesizes IGF-1 as a systemic growth factor. IGF-1 can also be synthesized by extrahepatic tissues, such as skeletal muscle, which affects its own synthesis through autocrine and paracrine. Many studies have found that IGF-1 oversecretion can maintain skeletal muscle quality and function ${ }^{40,41}$ and promote the synthesis of skeletal muscle cells. ${ }^{42,43}$ Furthermore, Brunk et al suggested that the vicious cycle of muscle atrophy involves the decline of muscle antioxidant capacity and the gut microbiota may maintain muscles' normal function in terms of antioxidant capacity and mitochondrial function. $^{44}$

Ebner $\mathrm{N}$ et al found that urolithin $\mathrm{A}$, a natural intestinal metabolite produced by host microbiota and metabolized by food conversion products, can restore mitochondria' function in muscles, thereby maintaining the normal progress of energy metabolism. ${ }^{45}$ This may provide a certain reference for future research on improving mitochondrial function associated with aging.

\section{Mechanism: Immune and Inflammation}

Systemic chronic inflammation is one of the most important factors associated with sarcopenia. ${ }^{46-48}$ AbouSamra $M$ et al found that wild mice and ordinary experimental mice have different gut microbiota. Experimental mice are more susceptible to external invasion and have a similar frontal immune response to humans than wild mice. ${ }^{50}$ This suggests that the difference in gut microbiota may have a regulatory effect on the immune system.

As a recurrent chronic gut inflammatory disease of the intestinal tract, inflammatory bowel disease(IBD) has obvious changes in the gut microbiota. In patients with IBD, the microbiota that produces butyrate is reduced. Butyrate is a SCFA that reduces intestinal inflammation, protects the intestinal barrier and regulates intestinal homeostasis. ${ }^{51}$ Increased intestinal permeability is one of the manifestations of the destruction of intestinal barrier 
function and a potential pathogenic factor for IBD. Furthermore, in IBD patients, E. Coli can interact with intestinal epithelial cells and induce inflammation, leading to excessive production of pro-inflammatory cytokines such as IL-1, TNF- $\alpha$, etc., thereby promoting intestinal inflammation and destroying the intestinal barrier. ${ }^{52,53}$ Studies reported that compared with normal people, IBD patients are more likely to have sarcopenia. The mechanisms underlying sarcopenia in IBD mainly include chronic intestinal inflammation, malabsorption of nutrients, lack of exercise,imbalance of gut microbiota ${ }^{54-56}$ Therefore, chronic intestinal inflammation, increased intestinal permeability and malnutrition in patients with IBD may affect skeletal muscle mass, leading to sarcopenia, in which the gut microbiota plays an important role.

Cancer cachexia refers to the severe weight, fat, and muscle loss of cancer patients, ${ }^{57-59}$ which is related to the increase of catabolism. Patients with cachexia have chronic inflammation and weakened immunity. ${ }^{54-56}$ In addition, many pathogenic factors of cachexia overlap with sarcopenia. Patients with advanced cancer are more likely to have both cachexia and sarcopenia, especially elderly cancer patients. ${ }^{60,61}$

The composition of gut microbiota is different in patients with cancer cachexia that in healthy individuals. ${ }^{62-64}$ Bindels et al found a decreased amount of Lactobacillus in mice with leukemia. Yet, after supplementing Lactobacillus to these mice with leukemia, the levels of inflammation factors such as interleukin-6, monocyte chemoattractant protein-1,interleukin-4 dropped. ${ }^{65}$ Another animal experiment found a lower number of lactobacillus and a higher amount of Enterobacteriaceae and Parabacteroides in mice with leukemia and colon cancer. After treating mice with Lactobacillus, cancer cell proliferation, as well as muscle loss and deficiency, were reduced. ${ }^{66}$ Pötgens and colleagues found an increased amount of Klebsiella oxytoca (a type of Enterobacter) in mice with cancer cachexia. This bacterium has been associated with a distributed balance of gut microbiota and the destruction of intestinal defensive barriers. ${ }^{67}$

To sum up, these studies suggest a strong relationship between gut microbiota and cancer cachexia. Supplementation with probiotics can reduce the cachexia state and inflammation. Interestingly, regular physical exercise can reduce inflammation in the body and promote health by changing the gut microbiota. ${ }^{68}$ Furthermore, it is known that autophagy is a key factor in the pathogenesis of SOB (sarcopenic obesity), a disease with a chronic inflammatory state. Autophagy can remove dysfunctional organelles in senescent cells, thereby retaining normal mitochondria and ensuring normal skeletal muscle quality. AMP-activated protein kinase (AMPK) and the peroxisome proliferator-activated receptor-gamma coactivator-1 alpha (PGC-1 $\alpha$ ) signaling pathways has been involved in the activation of autophagy. These signaling pathways also appear to be involved in the gut- muscle axis. Therefore, the gut microbiota disorder may be related to the onset of SOB. ${ }^{69}$

\section{Gut Microbiota: The Bridge Between Exercise and Sarcopenia}

Nutrition and exercise intervention are the most important interventions for sarcopenia. ${ }^{70-73}$ Nutritional supplements currently proven to be useful for sarcopenia are protein, ${ }^{74,75}$ essential amino acids, ${ }^{76}$ Vitamin D, bisphosphonates, calcifediol and calcium. Vitamin D supplementation has a small but significant effect on muscle strength, but the specific dosage, duration of use and the underlying mechanism have not been fully explained. ${ }^{77,78}$ Calcifediol has a significant effect on improving muscle function in postmenopausal women. ${ }^{79}$ The combined use of calcium and phosphate can significantly improve the muscle strength of patients with hip fractures. ${ }^{80}$ The role of protein in sarcopenia will be discussed in the next section.

Different types of exercise have different effects on muscles. Aerobic endurance exercise can increase the flexibility of exercise. Still, muscle strength and muscle mass improvement are not obvious and can only be used as an auxiliary exercise method. Resistance exercise can significantly improve muscle strength and affect muscle function and structure at the same time. ${ }^{70,71}$

Evans et al found different gut microbiota composition in obese mice and healthy animals. After exercise intervention, the gut microbiota of the experimental group was similar to the one found in normal mice. ${ }^{81}$ Zhao et al found that gut microbiota's composition (the feces' organic acids are significantly increased, and the nucleic acid-related substances are significantly reduced) drastically changes after a long endurance run (eg, half marathon) ${ }^{82}$ Exercise can also lead to the increase of muscle mass and function through gut microbiota, thereby reducing the occurrence of sarcopenia. Morita et al found that aerobic exercise 12-week training with brisk walking increased intestinal Bacteroides, as well as trunk muscle 
strength and cardiopulmonary function in elderly women (over the age of 65). ${ }^{83}$ This is due to the following reasons: firstly, exercise increases the blood flow in the guts, strengthens the contraction of abdominal muscles, and accelerates the release of gastrointestinal hormones, thereby speeding up the peristalsis of food and shortening the time to pass through the colon. Thus, the $\mathrm{pH}$ of the colon affects the gut microbiota. ${ }^{84-86}$ Secondly, aerobic exercise increases the content of short-chain fatty acids (SCFA) in the feces, which can slightly reduce the intestines' $\mathrm{pH}$, leading to very suitable conditions for intestinal Bacteroides growth. ${ }^{87,88}$ Intestinal probiotics can reduce inflammation, prevent diarrhea, and intestinal infections, all of which are favorable for the development of sarcopenia. $^{89}$

\section{Mechanism: Nutritional Supplements}

Protein supplementation is commonly used for the prevention and treatment of sarcopenia. ${ }^{70,72,73}$ Meat is one of the main sources of protein ${ }^{90}$ that can be classified into red meat (pork and beef), white meat (chicken and fish), and non-meat protein (soy and cereal). Zhu et $\mathrm{al}^{91}$ compared the composition of gut bacteria in the caecum obtained from rats fed with proteins from white meat (chicken and fish), red meat (beef and pork), and other sources (casein and soy). They found a higher amount of Lactobacillus in the white meat than in the red meat or non-meat protein groups.However, Ma et $\mathrm{al}^{92}$ found that Lactobacilli, Bifidobacteria, and butyrate-producing bacteria were reduced after protein supplementation in mice. This may be related to the difference in experimental methods.

Gut microbiota can affect the body's antioxidant effect, skeletal muscle health, and athletic ability. ${ }^{93}$ MorenoPérez et $\mathrm{al}^{94}$ found no increase in microbiota fermentation products (ammonia and SCFA concentrations) but an increase in the number of microbiota in athletes that received 10 weeks of protein supplementation (a mixture of whey and beef). This bacteria can fully decompose protein, increase intestinal protein's utilization rate, ${ }^{94}$ and use urea as a nitrogen source. ${ }^{95}$ In addition, the number of Blautia, Roseburia, and Bifidobacterium longum decreased; Blautia and Roseburia are associated with the production of SCFA, which has been proved to have health benefits. ${ }^{96}$ In addition, Blanton et al found immature gut microbiota in malnourished, which indicated that bacteria in the gut might be related to the growth and development of children. In contrast, the gut imbalance microbiota may cause difficulty in nutrient absorption. ${ }^{97}$
Newsome et al used isotopic and genetic methods to explore gut microbiota that can provide an essential source for the synthesis of host tissue structure: essential amino acids, of which valine is most absorbed. ${ }^{98}$ Valine and other branched-chain amino acids are the raw materials for protein synthesis and important energy sources in the body during hunger, lactation, exercise, and disease: the energy conversion of liver gluconeogenesis is accelerated, and the serum concentration of alanine and glutamic acid is reduced. At this time, supplementing branched-chain amino acids can save muscle glycogen, make the energy source of skeletal muscle to last, and increase muscle endurance. $^{99,100}$

Many studies have established that intestinal microbiota can produce short-chain fatty acids (SCFAs). ${ }^{94,96} \mathrm{In}$ mice treated with antibiotics, the gut microbiota, the number of SCFAs, and the exercise endurance are reduced. After adding SCFAs, the exercise endurance of mice significantly increases. Therefore, the gut microbiota may affect muscle energy metabolism through SCFAs. ${ }^{101}$ The gut microbiota can ferment indigestible carbohydrates to produce short-chain fatty acids. SCFS is mainly composed of three substances: acetate, propionate, and butyrate, absorbed from the intestinal cavity to regulate the metabolism of the whole body and affect muscle and fat metabolism. ${ }^{102}$ SCFS can also regulate energy metabolism and immunity, prevent and treat the occurrence of sarcopenia and muscle atrophy. ${ }^{103,104}$ The degree of fatty acid saturation is also highly correlated. In mice treated with saturated (HFD-sat),n-6 (HFD-n6), or n-3 (HFD-n3) fatty acids for 8 weeks, the saturated fatty acid group increased insulin resistance, colon permeability, and mesentery fat inflammation. ${ }^{105}$ Zou et al implemented a calorierestricted diet for non-obese patients $(40 \%$ less than the recommended daily intake) and found limited changes in gut microbiota were limited; the changes in the concentration of gut microbiota-dependent plasma amino acids: 3Increased methylhistidine, this substance is related to skeletal muscle protein. ${ }^{106}$ However, there is no research directly on the connection between sarcopenia and nutrition through the gut microbiota, although there have been speculations that indirectly confirm this.

Some studies found that alcohol, dietary fiber can play a role in the gut-muscle axis. A cross-sectional study discovered that a high fiber diet could lead to a better physical function (short physical performance battery (SPPB) score and grip strength) compared with a diet with low fibers. ${ }^{107}$ Bjørkhaug et al found a lower muscle 
mass and different gut microbiota (more proteobacteria and fewer Enterococcus faecalis bacteria) in people with a long history of heavy alcohol consumption compared to a control group. ${ }^{108}$ However, this study only clarified this phenomenon but did not prove the causality.

At the same time, another study found that eating time (day or night) has a certain impact on gut microbiota and muscle function. In comparison, eating at night should be avoided. Hormonal changes may play a role in this. ${ }^{109}$

Previous studies ${ }^{110}$ have found that metronidazole can upregulate the expression of factors related to skeletal muscle neurogenic atrophy, such as Hdac4, myogenin, MuRF1, and atrogin1, decreasing the muscle mass of their hind limbs and the muscle fiber volume of the anterior tibial muscle in mice. It can also cause changes in the gut microbiota, which in turn affect the expression of genes related to muscle metabolism, including Cry2, Ror- $\beta$, E4BP4, PPAR $\gamma$, Bmal1, Per2, FoxO1, and Pdk4, and the expression of adiponectin increased. Adiponectin $(\mathrm{ApN})$ is considered to be significantly associated with the occurrence of sarcopenia. A prospective cohort study by Huang et al indicated that an increased plasma ApN level in 434 elderly people is significantly associated with decreased physical activity. ${ }^{111}$ Therefore, it is reasonable to guess that antibiotics use may cause gut microbiota imbalance and thus reduce muscle mass.

\section{Endocrine Part of Gut Microbiota}

Skeletal muscle and adipose tissue are endocrine organs that secrete myokines and adipokines, impacting skeletal muscle. Suriano et al found that the gut microbiota can affect the skeletal muscle using myokines and adipokines metabolism. ${ }^{112}$ Transplanting gut microbiota can increase fat mass and leptin in germ-free mice. ${ }^{103}$ Leptin promotes fatty acid oxidation and triglyceride breakdown in skeletal muscle cells. Some studies believe that leptin can increase the risk of sarcopenia. It can also reflect inflammation related to lipodystrophy, thereby promoting sarcopenia through chronic inflammation. ${ }^{17,18}$ Higher serum content of adiponectin, which has anti-inflammatory, antioxidant, and insulin-sensitizing effects, has been found in mice treated with antibiotics. ${ }^{33}$ Furthermore, Huang et al found that the increase in plasma adiponectin levels in 434 elderly people was significantly associated with decreased physical activity and was associated with the occurrence of sarcopenia. ${ }^{34}$ This data indicate that the gut microbiota has an essential role in regulating different adipokines. Moreover, androgens have the effect of promoting muscle synthesis, and supplementing the gut microbiota can reduce muscle loss in individuals lacking androgens. ${ }^{113}$ Therefore, the gut microbiota can interact with the endocrine system related to muscle mass and sarcopenia.

In obese mice, certain gut microbiota and its metabolites can regulate insulin clearance, while in mice treated with antibiotics, insulin clearance is impaired. Clostridiaceae is related to insulin levels, Enterococcaceae correlated with impaired insulin clearance, and Peptostreptococcaceae is related to insulin secretion damage. Hyperinsulinemia is the cause and result of obesity or insulin resistance. Hyperinsulinemia comes from insufficient insulin clearance or excessive insulin production. Insulin resistance makes muscle cells unable to use glucose but can only use glycogen or fat, which may cause muscle mass loss and cause sarcopenia. ${ }^{36}$

\section{Conclusions: Gut Microbiota: The Neglected Actor in Sarcopenia?}

The gut microbiota can interact with muscle mass and its function through inflammation, immunity, energy metabolism, and insulin sensitivity, thus affecting the body's physiological function. The diagnostic criteria for sarcopenia and the detection methods of intestinal flora are rapidly developing, thus making the research process more convenient and efficient. Future research is warranted to further confirm the correlation, mechanism, and other details of the gut-muscle axis. Moreover, further studies on nutrition and exercise, and gut-muscle axis are also needed. Scholars should also focus on the effects of probiotics, prebiotics, synbiotics, and certain drugs on muscle mass and muscle function, as the gut-muscle axis is a very promising research direction in sarcopenia.

\section{Acknowledgments}

Supported by Shanghai Municipal Health Commission (201940415) and Shanghai Municipal Health Commission (202040301).

\section{Disclosure}

The authors report no conflicts of interest in this work.

\section{References}

1. Cruz-Jentoft AJ, Bahat G, Bauer J, et al. Sarcopenia: revised European consensus on definition and diagnosis. Age Ageing. 2019;48:16-31. 
2. Chen LK, Woo J, Assantachai P, et al. Asian working group for sarcopenia: 2019 consensus update on sarcopenia diagnosis and treatment. J Am Med Dir Assoc. 2020;21:300-307.

3. Shafiee G, Keshtkar A, Soltani A, Ahadi Z, Larijani B, Heshmat R. Prevalence of sarcopenia in the world: a systematic review and meta- analysis of general population studies. J Diabetes Metab Disord. 2017;16:21.

4. Fox B, Henwood T, Schaap L, et al. Adherence to a standardized protocol for measuring grip strength and appropriate cut-off values in adults over 65 years with sarcopenia: a systematic review protocol. JBI Database System Rev Implement Rep. 2015;13:50-59.

5. Van Ancum JM, Alcazar J, Meskers C, Nielsen BR, Suetta C, Maier AB. Impact of using the updated EWGSOP2 definition in diagnosing sarcopenia: a clinical perspective. Arch Gerontol Geriatr. 2020;90:104125.

6. Cruz-Jentoft AJ, Baeyens JP, Bauer JM, et al. Sarcopenia: european consensus on definition and diagnosis: report of the European Working Group on Sarcopenia in Older People. Age Ageing. 2010;39:412-423.

7. Lauretani F, Russo CR, Bandinelli S, et al. Age-associated changes in skeletal muscles and their effect on mobility: an operational diagnosis of sarcopenia. J Appl Physiol (1985). 2003;95:1851-1860.

8. Martone AM, Marzetti E, Salini S, et al. Sarcopenia identified according to the EWGSOP2 definition in community-living people: prevalence and clinical features. $J$ Am Med Dir Assoc. 2020.

9. Kim M, Won CW. Prevalence of sarcopenia in community-dwelling older adults using the definition of the European Working Group on Sarcopenia in Older People 2: findings from the Korean Frailty and Aging Cohort Study. Age Ageing. 2019;48:910-916.

10. Waltz TB, Fivenson EM, Morevati M, et al. Sarcopenia, aging and prospective interventional strategies. Curr Med Chem. 2018;25:5588-5596.

11. Liguori I, Russo G, Aran L, et al. Sarcopenia: assessment of disease burden and strategies to improve outcomes. Clin Interv Aging. 2018;13:913-927.

12. Chen J, Douglass J, Prasath V, et al. The microbiome and breast cancer: a review. Breast Cancer Res Treat. 2019;178:493-496.

13. Li J, Jia H, Cai X, et al. An integrated catalog of reference genes in the human gut microbiome. Nat Biotechnol. 2014;32:834-841.

14. Dave M, Higgins PD, Middha S, Rioux KP. The human gut microbiome: current knowledge, challenges, and future directions. Transl Res. 2012;160:246-257.

15. Jager R, Zaragoza J, Purpura M, et al. Probiotic administration increases amino acid absorption from plant protein: a placebo-controlled, randomized, double-blind, multicenter, crossover study. Probiotics Antimicrob Proteins. 2020.

16. Ford AC, Harris LA, Lacy BE, et al. Systematic review with meta-analysis: the efficacy of prebiotics, probiotics, synbiotics and antibiotics in irritable bowel syndrome. Aliment Pharmacol Ther. 2018;48(10):1044-1060. doi:10.1111/apt.15001

17. Mangiola F, Nicoletti A, Gasbarrini A, Ponziani FR. Gut microbiota and aging. Eur Rev Med Pharmacol Sci. 2018;22: 7404-7413.

18. Kim S, Jazwinski SM. The gut microbiota and healthy aging: a mini-review. Gerontology. 2018;64:513-520.

19. Seifi N, Safarian M, Nematy M, Rezvani R, Khadem-Rezaian M, Sedaghat A. Effects of synbiotic supplementation on energy and macronutrients homeostasis and muscle wasting of critical care patients: study protocol and a review of previous studies. Trials. 2020;21:221
20. Fielding RA, Reeves AR, Jasuja R, Liu C, Barrett BB, Lustgarten MS. Muscle strength is increased in mice that are colonized with microbiota from high-functioning older adults. Exp Gerontol. 2019;127:110722.

21. Buigues C, Fernandez-Garrido J, Pruimboom L, et al. Effect of a prebiotic formulation on frailty syndrome: a randomized, double-blind clinical trial. Int J Mol Sci. 2016;17.

22. Munukka E, Rintala A, Toivonen R, et al. Faecalibacterium prausnitzii treatment improves hepatic health and reduces adipose tissue inflammation in high-fat fed mice. ISME J. 2017;2017:24.

23. Lee MC, Hsu YJ, Ho HH, et al. Lactobacillus salivarius subspecies salicinius SA-03 is a new probiotic capable of enhancing exercise performance and decreasing fatigue. Microorganisms. $2020 ; 8$.

24. Lee MC, Hsu YJ, Chuang HL, et al. In vivo ergogenic properties of the bifidobacterium longum OLP-01 isolated from a weightlifting gold medalist. Nutrients. 2019;11.

25. Nay K, Jollet M, Goustard B, et al. bacteria are critical for optimal muscle function: a potential link with glucose homeostasis. Am J Physiol Endocrinol Metab. 2019;317:E158E171.

26. Ragul K, Kandasamy S, Devi PB, Shetty PH. Evaluation of functional properties of potential probiotic isolates from fermented brine pickle. Food Chem. 2020;311:126057.

27. Ragul K, Syiem I, Sundar K, Shetty PH. Characterization of probiotic potential of Bacillus species isolated from a traditional brine pickle. J Food Sci Technol. 2017;54:4473-4483.

28. Kazmierczak-Siedlecka K, Folwarski M, Skonieczna-Zydecka K, Ruszkowski J, Makarewicz W. The use of Lactobacillus plantarum 299v (DSM 9843) in cancer patients receiving home enteral nutrition - study protocol for a randomized, double-blind, and placebo-controlled trial. Nutr J. 2020;19:98.

29. Huang WC, Lee MC, Lee CC, et al. Effect of Lactobacillus plantarum TWK10 on exercise physiological adaptation, performance, and body composition in healthy humans. Nutrients. $2019 ; 11$.

30. Sun L, Li J, Lan LL, Li XA. The effect of fecal microbiota transplantation on Hepatic myelopathy: a case report. Medicine (Baltimore). 2019;98:e16430.

31. Chen YM, Wei L, Chiu YS, et al. Lactobacillus plantarum TWK10 supplementation improves exercise performance and increases muscle mass in mice. Nutrients. 2016;8(4):205.

32. Marzetti E, Calvani R, Cesari M, et al. Mitochondrial dysfunction and sarcopenia of aging: from signaling pathways to clinical trials. Int J Biochem Cell Biol. 2013;45:2288-2301.

33. Joseph AM, Adhihetty PJ, Buford TW, et al. The impact of aging on mitochondrial function and biogenesis pathways in skeletal muscle of sedentary high- and low-functioning elderly individuals. Aging Cell. 2012;11:801-809.

34. Coen PM, Jubrias SA, Distefano G, et al. Skeletal muscle mitochondrial energetics are associated with maximal aerobic capacity and walking speed in older adults. $J$ Gerontol a Biol Sci Med Sci. 2013;68:447-455.

35. Leduc-Gaudet JP, Picard M, St-Jean PF, et al. Mitochondrial morphology is altered in atrophied skeletal muscle of aged mice. Oncotarget. 2015;6:17923-17937.

36. Short KR, Vittone JL, Bigelow ML, Proctor DN, Nair KS. Age and aerobic exercise training effects on whole body and muscle protein metabolism. Am J Physiol Endocrinol Metab. 2004;286:E92-E101.

37. Kim Y, Triolo M, Hood DA. Impact of aging and exercise on mitochondrial quality control in skeletal muscle. Oxid Med Cell Longev. 2017;2017:3165396.

38. Franco-Obregon A, Gilbert JA. The microbiome-mitochondrion connection: common ancestries, common mechanisms, common goals. mSystems. 2017;2. 
39. Lahiri S, Kim H, Garcia-Perez I, et al. gut microbiota influences skeletal muscle mass and function in mice. Sci Transl Med. 2019;11.

40. Barton-Davis ER, Shoturma DI, Musaro A, Rosenthal N, Sweeney HL. Viral mediated expression of insulin-like growth factor I blocks the aging-related loss of skeletal muscle function. Proc Natl Acad Sci U S A. 1998;95:15603-15607.

41. Bosch-Marce M, Wee CD, Martinez TL, et al. 1 in muscle modulates the phenotype of severe SMA mice. Hum Mol Genet. 2011;20:1844-1853.

42. Brisson BK, Spinazzola J, Park S, Barton ER. Viral expression of insulin-like growth factor I E-peptides increases skeletal muscle mass but at the expense of strength. Am J Physiol Endocrinol Metab. 2014;306:E965-E974.

43. Abou-Samra M, Selvais CM, Boursereau R, Lecompte S, Noel L, Brichard SM. AdipoRon, a new therapeutic prospect for Duchenne muscular dystrophy. J Cachexia Sarcopenia Muscle. 2020 .

44. Brunk UT, Terman A. The mitochondrial-lysosomal axis theory of aging: accumulation of damaged mitochondria as a result of imperfect autophagocytosis. Eur $J$ Biochem. 2002;269:19 96-2002.

45. Ebner N, Anker SD, von Haehling S. Recent developments in the field of cachexia, sarcopenia, and muscle wasting: highlights from the 11th Cachexia Conference. J Cachexia Sarcopenia Muscle. 2019; 10:218-225.

46. Wilson D, Jackson T, Sapey E, Lord JM. Frailty and sarcopenia: the potential role of an aged immune system. Ageing Res Rev. 2017;36:1-10.

47. Yoo JI, Ha YC, Choi H, et al. Malnutrition and chronic inflammation as risk factors for sarcopenia in elderly patients with hip fracture. Asia Pac J Clin Nutr. 2018;27:527-532.

48. Byun MK, Cho EN, Chang J, Ahn CM, Kim HJ. Sarcopenia correlates with systemic inflammation in COPD. Int $J$ Chron Obstruct Pulmon Dis. 2017;12:669-675.

49. Abou-Samra M, Selvais CM, Boursereau R, Lecompte S, Noel L, Brichard SM. AdipoRon, a new therapeutic prospect for Duchenne muscular dystrophy. J Cachexia Sarcopenia Muscle. 2020 .

50. Mentella MC, Scaldaferri F, Pizzoferrato M, et al. Nutrition, IBD and Gut Microbiota: a Review. Nutrients. 2020;12:4.

51. Graziani C, Talocco C, De Sire R, et al. Intestinal permeability in physiological and pathological conditions: major determinants and assessment modalities. Eur Rev Med Pharmacol Sci. 2019;23(2):795-810.

52. De Sire R, Talocco C, Petito V, et al. [Microbiota and inflammatory bowel disease: an update]. Recenti Prog Med. 2018;109 (12):570-573. [Article in Italian].

53. Pizzoferrato M, de Sire R, Ingravalle F, et al. Characterization of Sarcopenia in an IBD population attending an Italian Gastroenterology Tertiary Center. Nutrients. 2019;11:10.

54. Scaldaferri F, Pizzoferrato M, Lopetuso LR, et al. Nutrition and IBD: malnutrition and/or sarcopenia? A practical guide. Gastroenterol Res Pract. 2017;2017:8646495.

55. de Sire R, Rizzatti G, Ingravalle F, et al. Skeletal muscle-gut axis: emerging mechanisms of sarcopenia for intestinal and extra intestinal diseases. Minerva Gastroenterol Dietol. 2018;64 (4):351-362.

56. Picca A, Ponziani FR, Calvani R, et al. Gut microbial, inflammatory and metabolic signatures in older people with physical frailty and sarcopenia: results from the BIOSPHERE study. Nutrients. $2019 ; 12$.

57. Siddharth J, Chakrabarti A, Pannerec A, et al. Aging and sarcopenia associate with specific interactions between gut microbes, serum biomarkers and host physiology in rats. Aging (Albany NY). 2017;9:1698-1720.
58. Gophna U, Konikoff T, Nielsen HB. Oscillospira and related bacteria - From metagenomic species to metabolic features. Environ Microbiol. 2017;19:835-841.

59. Padoan A, Plebani M, Basso D. Inflammation and pancreatic cancer: focus on metabolism, cytokines, and immunity. Int J Mol Sci. 2019;20.

60. Tuomisto AE, Makinen MJ, Vayrynen JP. Systemic inflammation in colorectal cancer: underlying factors, effects, and prognostic significance. World J Gastroenterol. 2019;25:4383-4404.

61. Dev R, Wong A, Hui D, Bruera E. The evolving approach to management of cancer cachexia. Oncology (Williston Park). 2017;31:23-32.

62. Ali S, Garcia JM. Sarcopenia, cachexia and aging: diagnosis, mechanisms and therapeutic options - a mini-review. Gerontology. 2014;60:294-305.

63. Dunne RF, Loh KP, Williams GR, Jatoi A, Mustian KM, Mohile SG. Cachexia and sarcopenia in older adults with cancer: a comprehensive review. Cancers (Basel). 2019;11.

64. Bindels LB, Beck R, Schakman O, et al. Restoring specific lactobacilli levels decreases inflammation and muscle atrophy markers in an acute leukemia mouse model. PLoS One. 2012;7: e37971.

65. Bindels LB, Neyrinck AM, Claus SP, et al. Synbiotic approach restores intestinal homeostasis and prolongs survival in leukaemic mice with cachexia. Isme J. 2016;10:1456-1470.

66. Potgens SA, Brossel H, Sboarina M, et al. Klebsiella oxytoca expands in cancer cachexia and acts as a gut pathobiont contributing to intestinal dysfunction. Sci Rep. 2018;8:12321.

67. Cerda B, Perez M, Perez-Santiago JD, Tornero-Aguilera JF, Gonzalez-Soltero R, Larrosa M. Gut microbiota modification: another piece in the puzzle of the benefits of physical exercise in health? Front Physiol. 2016;7:51.

68. J Y R, H M C, H I Y, et al. Dysregulated autophagy mediates sarcopenic obesity and its complications via AMPK and PGC1alpha signaling pathways: potential involvement of gut dysbiosis as a pathological link. Int J Mol Sci. 2020;21:18.

69. Maltais ML, Ladouceur JP, Dionne IJ. The effect of resistance training and different sources of postexercise protein supplementation on muscle mass and physical capacity in sarcopenic elderly men. J Strength Cond Res. 2016;30:1680-1687.

70. Strasser B, Keinrad M, Haber P, Schobersberger W. Efficacy of systematic endurance and resistance training on muscle strength and endurance performance in elderly adults-a randomized controlled trial. Wien Klin Wochenschr. 2009;121:75 7-764.

71. Martone AM, Marzetti E, Calvani R, et al. Exercise and protein intake: a synergistic approach against sarcopenia. Biomed Res Int. 2017;2017:2672435.

72. Dhillon RJ, Hasni S. Pathogenesis and management of sarcopenia. Clin Geriatr Med. 2017;33:17-26.

73. Landi F, Calvani R, Tosato $\mathrm{M}$, et al. Protein intake and muscle health in old age: from biological plausibility to clinical evidence. Nutrients. 2016;8:5.

74. Marshall RN, Smeuninx B, Morgan PT, et al. Nutritional strategies to offset disuse-induced skeletal muscle atrophy and anabolic resistance in older adults: from whole-foods to isolated ingredients. Nutrients. 2020;12:5.

75. Invernizzi M, de Sire A, D'Andrea F, et al. Effects of essential amino acid supplementation and rehabilitation on functioning in hip fracture patients: a pilot randomized controlled trial. Aging Clin Exp Res. 2019;31(10):1517-1524.

76. Beaudart C, Buckinx F, Rabenda V, et al. The effects of vitamin D on skeletal muscle strength, muscle mass, and muscle power: a systematic review and meta-analysis of randomized controlled trials. J Clin Endocrinol Metab. 2014;99 (11):4336-4345 
77. Gimigliano F, Moretti A, de Sire A, et al. The combination of vitamin D deficiency and overweight affects muscle mass and function in older post-menopausal women. Aging Clin Exp Res. 2018;30(6):625-631.

78. Iolascon G, Moretti A, de Sire A, et al. Effectiveness of calcifediol in improving muscle function in post-menopausal women: a prospective cohort study. Adv Ther. 2017;34(3):744-752.

79. Avola M, Mangano G, Testa G, et al. Rehabilitation strategies for patients with femoral neck fractures in sarcopenia: a narrative review. J Clin Med. 2020;9:10.

80. Evans CC, LePard KJ, Kwak JW, et al. Exercise prevents weight gain and alters the gut microbiota in a mouse model of high fat diet-induced obesity. PLoS One. 2014;9:e92193.

81. Zhao X, Zhang Z, Hu B, Huang W, Yuan C, Zou L. Response of gut microbiota to metabolite changes induced by endurance exercise. Front Microbiol. 2018;9:765.

82. Morita E, Yokoyama H, Imai D, et al. Aerobic exercise training with brisk walking increases intestinal bacteroides in healthy elderly women. Nutrients. 2019;11.

83. Tottey W, Feria-Gervasio D, Gaci N, et al. Colonic transit time is a driven force of the gut microbiota composition and metabolism: in vitro evidence. J Neurogastroenterol Motil. 2017;23:124-134.

84. Abbas A, Wilding GE, Sitrin MD. Does colonic transit time affect colonic pH?. J Gastroenterol Hepatol Res. 2014;3:1103-1107.

85. De Schryver AM, Keulemans YC, Peters HP, et al. Effects of regular physical activity on defecation pattern in middle-aged patients complaining of chronic constipation. Scand J Gastroenterol. 2005;40:422-429.

86. Matsumoto $\mathrm{M}$, Inoue $\mathrm{R}$, Tsukahara $\mathrm{T}$, et al. Voluntary running exercise alters microbiota composition and increases n-butyrate concentration in the rat cecum. Biosci Biotechnol Biochem. 2008;72:572-576.

87. Walker AW, Duncan SH, McWilliam LE, Child MW, Flint HJ. pH and peptide supply can radically alter bacterial populations and short-chain fatty acid ratios within microbial communities from the human colon. Appl Environ Microbiol. 2005;71:3692-3700.

88. Vlasova AN, Kandasamy S, Chattha KS, Rajashekara G, Saif LJ. Comparison of probiotic lactobacilli and bifidobacteria effects, immune responses and rotavirus vaccines and infection in different host species. Vet Immunol Immunopathol. 2016;172:72-84.

89. Damanti S, Azzolino D, Roncaglione C, Arosio B, Rossi P, Cesari M. Efficacy of nutritional interventions as stand-alone or synergistic treatments with exercise for the management of sarcopenia. Nutrients. 2019;11.

90. Zhu Y, Lin X, Zhao F, et al. Erratum: meat, dairy and plant proteins alter bacterial composition of rat gut bacteria. Sci Rep. 2015;5:16546.

91. Ma N, Tian Y, Wu Y, Ma X. Contributions of the interaction between dietary protein and gut microbiota to intestinal health. Curr Protein Pept Sci. 2017;18:795-808.

92. Dai X, Karring H. A determination and comparison of urease activity in feces and fresh manure from pig and cattle in relation to ammonia production and pH changes. PLoS One. 2014;9:e110402.

93. Moreno-Perez D, Bressa C, Bailen M, et al. Effect of a Protein Supplement on the Gut Microbiota of Endurance Athletes: A Randomized, Controlled, Double-Blind Pilot Study[J]. Nutrients. 2018,10(3).

94. Yatsunenko T, Rey FE, Manary MJ, et al. Human gut microbiome viewed across age and geography. Nature. 2012;486:222-227.

95. Moreno-Perez D, Bressa C, Bailen M, et al. Effect of a protein supplement on the gut microbiota of endurance athletes: a randomized, controlled, double-blind pilot study. Nutrients. $2018 ; 10$.
96. Blanton LV, Charbonneau MR, Salih T, et al. Gut bacteria that prevent growth impairments transmitted by microbiota from malnourished children. Science. 2016;351.

97. Newsome SD, Feeser KL, Bradley CJ, Wolf C, Takacs-Vesbach $\mathrm{C}$, Fogel ML. Isotopic and genetic methods reveal the role of the gut microbiome in mammalian host essential amino acid metabolism. Proc Biol Sci. 2020;287:20192995.

98. Wolfe RR. Branched-chain amino acids and muscle protein synthesis in humans: myth or reality? J Int Soc Sports Nutr. 2017;14:30.

99. Santos CS, Nascimento F. Isolated branched-chain amino acid intake and muscle protein synthesis in humans: a biochemical review. Einstein (Sao Paulo). 2019;17:B4898.

100. Okamoto T, Morino K, Ugi S, et al. Microbiome potentiates endurance exercise through intestinal acetate production. $\mathrm{Am}$ J Physiol Endocrinol Metab. 2019;316:E956-E966.

101. Frampton J, Murphy KG, Frost G, Chambers ES. Short-chain fatty acids as potential regulators of skeletal muscle metabolism and function. Nat Metab. 2020;2:840-848.

102. Hu J, Lin S, Zheng B, Cheung P. Short-chain fatty acids in control of energy metabolism. Crit Rev Food Sci Nutr. 2018; 58:1243-1249.

103. Scheithauer TP, Dallinga-Thie GM, de Vos WM, Nieuwdorp M, van Raalte DH. Causality of small and large gut microbiota in weight regulation and insulin resistance. Mol Metab. 2016;5:759-770.

104. Lam YY, Ha CW, Hoffmann JM, et al. Effects of dietary fat profile on gut permeability and microbiota and their relationships with metabolic changes in mice. Obesity (Silver Spring). 2015;23:1429-1439.

105. Zou H, Wang D, Ren H, et al. Effect of caloric restriction on BMI, gut microbiota, and blood amino acid levels in non-obese adults. Nutrients. 2020;12.

106. Barger K, Langsetmo L, Orwoll ES, Lustgarten MS. Investigation of the diet-gut-muscle axis in the osteoporotic fractures in men study. J Nutr Health Aging. 2020;24:445-452.

107. Bjorkhaug ST, Aanes H, Neupane SP, et al. Characterization of gut microbiota composition and functions in patients with chronic alcohol overconsumption. Gut Microbes. 2019;10:663-675.

108. Zhang L, Xue X, Zhai R, et al. Timing of calorie restriction in mice impacts host metabolic phenotype with correlative changes in gut microbiota. mSystems. 2019;4:6. doi:10.1128/ mSystems.00348-19.

109. Manickam R, Oh H, Tan CK, Paramalingam E, Wahli W. Metronidazole causes skeletal muscle atrophy and modulates muscle chronometabolism. Int J Mol Sci. 2018;19.

110. Huang C, Tomata Y, Kakizaki M, et al. High circulating adiponectin levels predict decreased muscle strength among older adults aged 70 years and over: a prospective cohort study. Nutr Metab Cardiovasc Dis. 2015;25:594-601.

111. Suriano F, Van Hul M, Cani PD. Gut microbiota and regulation of myokine-adipokine function. Curr Opin Pharmacol. 2020; 52:9-17.

112. Harada N, Hanaoka R, Hanada K, Izawa T, Inui H, Yamaji R. Hypogonadism alters cecal and fecal microbiota in male mice. Gut Microbes. 2016;7:533-539.

113. Foley KP, Zlitni S, Duggan BM, et al. Gut microbiota impairs insulin clearance in obese mice. Mol Metab. 2020;42:101067. 


\section{Publish your work in this journal}

The International Journal of General Medicine is an international, peer-reviewed open-access journal that focuses on general and internal medicine, pathogenesis, epidemiology, diagnosis, monitoring and treatment protocols. The journal is characterized by the rapid reporting of reviews, original research and clinical studies across all disease areas. The manuscript management system is completely online and includes a very quick and fair peer-review system, which is all easy to use. Visit http://www.dovepress.com/ testimonials.php to read real quotes from published authors.

Submit your manuscript here: https://www.dovepress.com/international-journal-of-general-medicine-journal 